\title{
Anstiftung zu mutiger Schulentwicklung
}

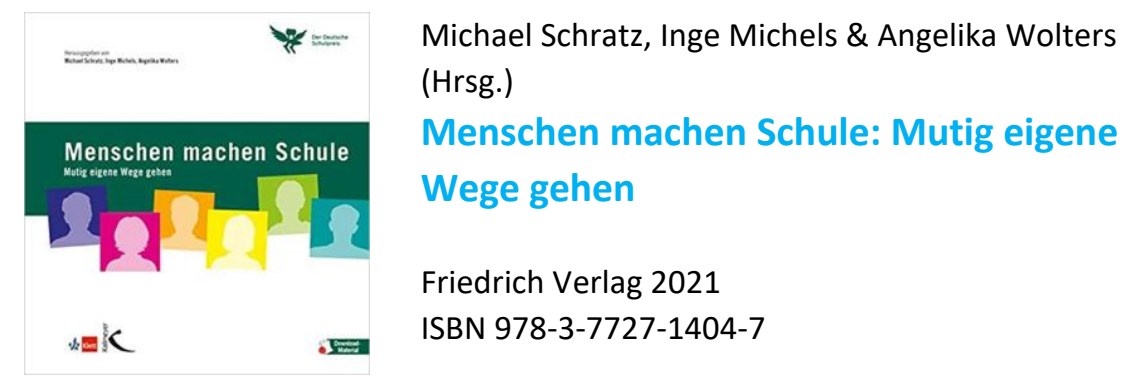

Akteur*innen des Bildungswesens aller Systemebenen haben die aktuell herausfordernde Situation zum Anlass genommen, Unterricht und Schulqualität zu reflektieren, geübte Handlungsweisen zu hinterfragen und neue Wege zu beschreiten. Sie haben die Corona-Krise entgegen aller Beharrungstendenzen als Chance und Anstoß für Weiterentwicklung gesehen und wichtige Entwicklungsschritte innerhalb kurzer Zeit umgesetzt. „Der gesellschaftliche Trend zur zunehmenden Digitalisierung und Automatisierung war auch schon vor der Pandemie nicht aufzuhalten" (S. 15), aber nun konnte bei der Nutzung digitaler Werkzeuge und Kommunikationsformen ein enormer Entwicklungssprung erreicht werden. Dass kurzfristig gesetzte Maßnahmen das Potenzial haben, langfristige und nachhaltige Veränderungen im Bildungssystem zu ermöglichen, zeigen die Beiträge im Sammelband „Menschen machen Schule: Mutig eigene Wege gehen“. Hier werden agile Schulen vor den Vorhang gebeten, die flexibel und individuell mit gesellschaftlichen und aktuellen Herausforderungen umgegangen sind und durch ihr Verständnis von Autonomie eigene, gleichermaßen innovative wie kreative Lösungen gefunden haben.

Wie entsteht eine lernförderliche, zu-mutende Praxis in der Schule? Spillane (2013) meint, im Dazwischen: Praxis entsteht situiert zwischen den Menschen, aber auch zwischen ihnen und den Werkzeugen, Routinen und Strukturen, die sich etabliert haben. „Menschen machen Schule" zeigt auf, wie diese auch aussehen können: Wenn sich z.B. die Leistungsbeurteilung in den Dienst des Lernens stellt (S. 176), wenn Mangel sich als Mehrwert herausstellt (S. 280) oder die Frage der Schul-Kultur über das Kulturelle hinausreicht (S. 80). 
Der rote Faden durch die vier Themencluster des Hauptteils ist der Mut. Da haben sich Schulmacher*innen mal was getraut: andersrum zu denken, zu strukturieren, auszuprobieren und zu etablieren. Deren Erfahrungen aus den Transformationsprozessen aktueller gesellschaftlicher Herausforderungen hinein in Schule und Unterricht widmen sich die Praxisberichte aus dem Kontext des Deutschen Schulpreises. Oft schwingt in den Interviews der jeweiligen Themengebenden das eigene Erstaunen ob des Erfolges mit. Und immer die Freude, dass es gelungen ist, Schule näher ans Kind, näher an den Menschen zu bringen. In fünfzehn Szenen „guter Schule“ begleiten wir, ähnlich einem Kaleidoskop, Schulmacher*innen bei ihren Einsichten und best failures, bei Durchbrüchen und Rückschlägen, bei lessons learnt und bei ihren nächsten Schritten von good zu next practice. Zu dieser möchte das Buch auch anregen: Nicht zum „Umsetzen“, sondern zum Hineinspüren, was passen könnte für den jeweils eigenen Standort. Durch Fragen zum Vor-Denken, Reflektieren und Diskutieren möchten die Autor*innen einladen, in die eigene Positionierung zum jeweiligen Thema zu gehen: Wie stehe ich z.B. zu Schüler*innen-Partizipation, heterogenitätssensiblem Unterricht und selbstbestimmtem Lernen? Um daraus dann Mut zu schöpfen für die eigenen Handlungsoptionen.

Die unterschiedlichen Zugänge verdeutlichen, dass menschliches Zusammenleben - sei es beruflich oder privat - immer von Haltung geprägt ist, die im gemeinsamen Tun sichtbar wird. Schratz betont in seinen zusammenfassenden Ausführungen die vielseitigen und facettenreichen Erscheinungsweisen von Haltung (S. 314), die auch in den unterschiedlichen Perspektiven der Interviewpartner*innen zum Ausdruck kommen. Lassen Sie sich von den Erfahrungsberichten und Fallbeispielen zu Reflexion und Wandel inspirieren - um eigene Wege zu beschreiten, getragen von Mut und positiver Haltung.

\section{Empfohlen von Christoph Hofbauer und Michaela Tscherne}

Spillane, J. (2013). The practice of leading and managing teaching in educational organisations. In OECD (2013). Leadership for $21^{\text {st }}$ Century Learning. Educational Research and Innovation. OECD Publishing. p. 59-82. 